MATHEMATICS OF COMPUTATION

Volume 70, Number 235, Pages 1265-1280

S $0025-5718(00) 01220-5$

Article electronically published on March 14, 2000

\title{
GEOMETRY OF THE JANTZEN REGION IN LUSZTIG'S CONJECTURE
}

\author{
BRIAN D. BOE
}

\begin{abstract}
The Lusztig Conjecture expresses the character of a finite-dimensional irreducible representation of a reductive algebraic group $G$ in prime characteristic as a linear combination of characters of Weyl modules for $G$. The representations described by the conjecture are in one-to-one correspondence with the (finitely many) alcoves in the intersection of the dominant cone and the so-called Jantzen region. Each alcove has a length, defined to be the number of alcove walls (hyperplanes) separating it from the fundamental alcove (the unique alcove in the dominant cone whose closure contains the origin). This article determines the maximum length of an alcove in the intersection of the dominant cone with the Jantzen region.
\end{abstract}

\section{INTRODUCTION}

The Lusztig Conjecture 6 expresses the character of a finite-dimensional irreducible representation $L_{w}$ of a reductive algebraic group $G$ in characteristic $p$ as a linear combination of characters of Weyl modules for $G$. The parameters $w$ live in the affine Weyl group $W_{p}$, an infinite group; this (at first glance) makes actual verification of the Lusztig Conjecture for any given group look like an "infinite" problem. However, the only w's to which the conjecture actually applies are those for which $w \rho$ is dominant and in the Jantzen region

$$
J=\left\{\lambda \in X(T) \otimes_{\mathbb{Z}} \mathbb{R} \mid\left(\lambda, \check{\alpha}_{0}\right) \leq p(p-h+2)\right\},
$$

where $X(T)$ denotes the group of characters of a maximal torus $T$ of $G, \rho$ is half the sum of the positive roots, $\check{\alpha}_{0}$ is the highest coroot, and $h$ is the Coxeter number of $G$. Geometrically, the relevant $w$ 's are in one-to-one correspondence with the alcoves lying in the intersection of the dominant cone and the Jantzen region, a bounded set in $X=X(T) \otimes_{\mathbb{Z}} \mathbb{R}$. In particular, the conjecture actually applies only to a finite set of $w$ 's; call this set $L$.

Some questions naturally arise:

1. What is the cardinality of $L$ ?

2. How many elements of $W_{p}$ must be computed before all elements of $L$ are certain to have appeared?

3. What is the maximum length of an element of $L$ ?

The first question is quite easy to answer (see Lemma 2.1). But if one were programming a computer to check a particular case of the Lusztig Conjecture, one would like to be able to provide the answer to the second question as input. The

Received by the editor May 22, 1998 and, in revised form, July 6, 1999.

2000 Mathematics Subject Classification. Primary 20G05; Secondary 20F55, 51F15.

(C)2000 American Mathematical Society 


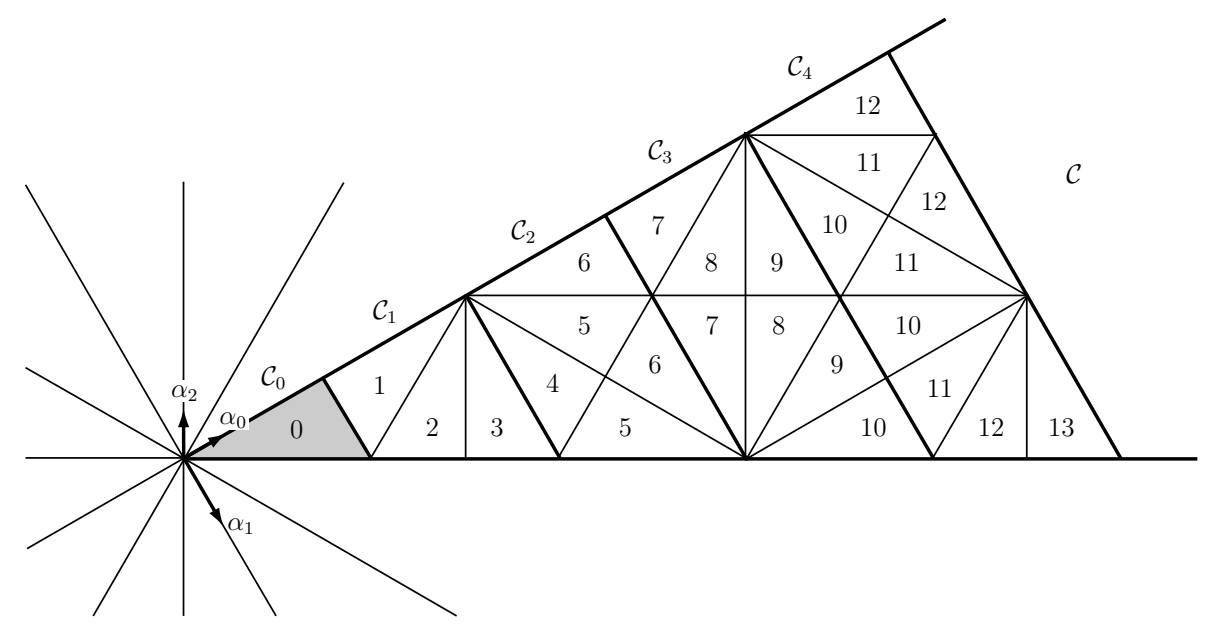

FiguRE 1. Geometry of the affine Weyl group of type $G_{2}$

cardinality of $L$ seems to provide very little information about the answer to the second question. On the other hand, an answer to the third question would be almost as useful, from the point of view of predicting in advance how much computation is necessary. It also turns out to be an interesting geometric and combinatorial problem. It is this last question which will be answered in the present article. (See [8] for a survey on the current status of the Lusztig Conjecture itself.)

More precisely (see Section 2 for unexplained notation), let $\mathcal{C}$ be the dominant cone

$$
\mathcal{C}=\left\{\lambda \in X \mid(\lambda, \check{\alpha})>0 \text { for all } \alpha \in R^{+}\right\}
$$

and define the $k^{\text {th }}$ level $(k=0,1,2, \ldots)$

$$
\mathcal{C}_{k}=\left\{\lambda \in C \mid k p<\left(\lambda, \check{\alpha}_{0}\right)<(k+1) p\right\} .
$$

An alcove is a set of the form

$$
A=\left\{\lambda \in X \mid k_{\alpha} p<(\lambda, \check{\alpha})<\left(k_{\alpha}+1\right) p \text { for all } \alpha \in R^{+}\right\}
$$

for certain integers $k_{\alpha}$, and the standard alcove $A_{0}$ is obtained by setting all $k_{\alpha}=0$. The alcoves are the connected components of $X-\cup \mathcal{H}$, where $\mathcal{H}$ is the collection of hyperplanes

$$
H_{\alpha, k}=\{\lambda \mid(\lambda, \check{\alpha})=k p\}, \quad \alpha \in R, k \in \mathbb{Z} .
$$

It is well known that $W_{p}$ permutes the alcoves simply transitively and, for $w \in W_{p}$, the length $\ell(w)$ of $w$ is equal to the number of hyperplanes $H \in \mathcal{H}$ such that $w A_{0}$ and $A_{0}$ are on opposite sides of $H$ (cf. [3]); set $\ell\left(w A_{0}\right)=\ell(w)$.

In Figure 1 we show the geometry associated to the affine Weyl group of type $G_{2}$ : the simple roots $\alpha_{1}$ and $\alpha_{2}$, and the highest short root $\alpha_{0}$; the root hyperplanes $H_{\alpha, 0}, \alpha \in R$; the standard alcove $A_{0}$ (shaded); levels 0 through 4 of the cone $\mathcal{C}$; and the lengths of the alcoves in those levels.

Our main theorem computes the maximum $\ell(w)$ for $w A_{0} \subset \mathcal{C}_{k}$. In the theorem, $p$ may be any positive integer (not necessarily prime). 
TABLE 1. Maximum length in level $k$

\begin{tabular}{|c|c|c|}
\hline Type of $G$ & $k$ even & $k$ odd \\
\hline$A_{n}$ & & $\frac{(n+1)^{2}}{4}$ \\
\hline$\overline{B_{n}}$ & \multicolumn{2}{|c|}{$k\left(\begin{array}{c}n+1 \\
2\end{array}\right)$} \\
\hline$C_{n} \quad n \leq 3$ & \multicolumn{2}{|c|}{$k(2 n-1)$} \\
\hline$n \geq 4$ & $k \frac{n^{2}}{2}$ & $k \frac{n^{2}}{2}-\frac{n}{2}+1$ \\
\hline$D_{n}, n \geq 4$ & \multicolumn{2}{|r|}{$k\left(\begin{array}{l}n \\
2\end{array}\right)$} \\
\hline$n=6$ & \multicolumn{2}{|r|}{$16 k$} \\
\hline$E_{n} \quad n=7$ & \multicolumn{2}{|r|}{$27 k$} \\
\hline$n=8$ & $46 k$ & $46 k-2-8 \delta_{k 1}$ \\
\hline$F_{4}$ & $11 k$ & $11 k-2$ \\
\hline$G_{2}$ & \multicolumn{2}{|r|}{$\left\lfloor\frac{10 k}{3}\right.$} \\
\hline
\end{tabular}

Theorem 1.1. Let $W_{p}$ be an affine Weyl group associated to a simply connected almost simple algebraic group $G$ defined over $\mathbb{Z}$. Let $\mathcal{C}_{k}$ and $A_{0}$ be as above. For each nonnegative integer $k$, the maximum $\ell(w), w \in W_{p}$, for which $w A_{0} \subset \mathcal{C}_{k}$ is given in Table 1 .

Since (for $p$ sufficiently large) $L=\left\{w \in W_{p} \mid w \rho \in \mathcal{C} \cap J\right\}=\left\{w \in W_{p} \mid w A_{0} \subset\right.$ $\mathcal{C}_{k}$ for some $\left.0 \leq k \leq p-h+1\right\}$, we immediately obtain the answer to Question 3 .

Corollary 1.2. Let $G$ be a simply-connected almost-simple algebraic group over a field of characteristic $p$, and $W_{p}$ the affine Weyl group of $G$; assume that $p \geq h$, the Coxeter number for $G$. Then the maximum length of a parameter $w$ to which the Lusztig Conjecture for $G$ applies, $\max \left\{\ell(w) \mid w \in W_{p}, w A_{0} \subset \mathcal{C} \cap J\right\}$, is given in Table $\mathbf{2}$.

TABle 2. Maximum length in the Jantzen region

\begin{tabular}{|c|c|c|}
\hline Tyl & e of $G$ & Maximum length \\
\hline & $A_{n}$ & \begin{tabular}{l|l}
$(p-n)$ & $\frac{(n+1)^{2}}{4}$
\end{tabular} \\
\hline & $B_{n}$ & $(p-2 n+1)\left(\begin{array}{c}n+1 \\
2\end{array}\right)$ \\
\hline & $n \leq 3$ & $(p-2 n+1)(2 n-1)$ \\
\hline & $n \geq 4$ & $(p-2 n+1) \frac{n^{2}}{2}$ \\
\hline$D_{n}$ & $n \geq 4$ & $(p-2 n+3)\left(\begin{array}{l}n \\
2\end{array}\right)$ \\
\hline & $n=6$ & $16(p-11)$ \\
\hline$E_{n}$ & $n=7$ & $27(p-17)$ \\
\hline & $n=8$ & $46(p-29)$ \\
\hline & $F_{4}$ & $11(p-11)$ \\
\hline & $G_{2}$ & $\frac{10(p-5)}{3}$ \\
\hline
\end{tabular}


Proof. Substitute $k=p-h+1$ in Table 1, along with the known values $h=n+1$, $2 n, 2 n, 2(n-1), 12,18,30,12,6$ in types $A_{n}, B_{n}, C_{n}, D_{n}, E_{6}, E_{7}, E_{8}, F_{4}, G_{2}$ respectively (cf. e.g. [3]), and use the fact that (except in type $A_{n}$ ) $p$ is odd and $h$ is even, thus $k$ is even.

Notice that the group $G$ and its representations really play no direct role in Theorem 1.1, it is actually a theorem about the geometry of affine Weyl groups. In the next section we will approach the problem from this more elementary point of view, and prove some general preliminary results. The remaining sections are devoted to proving the theorem in each of the various types.

\section{Notation AND Generalities}

We write $\mathbb{N}$ as usual for the set of positive integers, and $\mathbb{Z}_{+}$for the set of nonnegative integers. For $a \in \mathbb{R}$ and $n \in \mathbb{N}$, write $a(\bmod n)$ for the remainder when $a$ is divided by $n$; i.e., $a=n q+[a(\bmod n)]$, where $q \in \mathbb{Z}$ and $0 \leq a(\bmod n)<n$. By a half-integer we shall mean an element of $\mathbb{Z}+\frac{1}{2}$.

Let $\mathcal{A}$ be an $n_{0}$-dimensional affine Euclidean space with $n_{0} \geq 1$, and $\mathcal{H}$ a set of hyperplanes in $\mathcal{A}$. The connected components of $\mathcal{A}-\cup \mathcal{H}$ are called alcoves. For $H \in \mathcal{H}$ write $s_{H}$ for the orthogonal reflection of $\mathcal{A}$ with fixed point set $H$, and let $W_{\mathcal{H}}$ be the group generated by the reflections $s_{H}$. Write $X$ for the set of translations (i.e. vectors) in $\mathcal{A}$. Then $X \simeq \mathbb{R}^{n_{0}}$, and $W_{\mathcal{H}}$ acts naturally on $X$. Assume that $W_{\mathcal{H}}$ is an infinite discrete subgroup of the group of affine motions of $\mathcal{A}$ leaving $\mathcal{H}$ stable, and that the action of $W_{\mathcal{H}}$ on $X$ is irreducible. It follows from 2] that $W_{\mathcal{H}}$ is an affine Weyl group associated to an irreducible root system $R$ of rank $n_{0}$, and each alcove is an open simplex with $n_{0}+1$ faces. We can therefore define a dominant cone $\mathcal{C}$, levels $\mathcal{C}_{k}$, and fundamental alcove $A_{0}$ in this context, and pose Question 3 (see also [5]).

Equivalently, beginning instead with an irreducible root system $R$ of rank $n_{0}$ in a Euclidean space $X$, we can construct its associated affine Weyl group concretely as follows. For $\alpha \in R$ let $s_{\alpha}$ be the reflection through $\alpha$ acting on $X$. Fix a system $R^{+}$of positive roots, $\Delta$ the corresponding set of simple roots, and $W$ the Weyl group of $R$. Let $h$ be the Coxeter number of $R, \rho$ half the sum of the positive roots, and $\alpha_{0}$ the highest short root of $R$ (this makes $\check{\alpha}_{0}$ the highest coroot). Write $\langle\lambda, \alpha\rangle=2(\lambda, \alpha) /(\alpha, \alpha)=(\lambda, \check{\alpha})$, for $\lambda \in X, \alpha \in R$. Fix a positive integer $p$ (which need not be prime). For $k \in \mathbb{Z}$ let $H_{\alpha, k}$ be the hyperplane defined in (1.5), and $s_{\alpha, k}$ the affine reflection in $H_{\alpha, k}$; i.e., $s_{\alpha, k}(\lambda)=\lambda-(\langle\lambda, \alpha\rangle-k p) \alpha, \lambda \in X$. Then the affine Weyl group $W_{p}$ is the group generated by all $s_{\alpha, k}, \alpha \in R, k \in \mathbb{Z}$.

Recall the definitions of the dominant cone $\mathcal{C}$, the standard alcove $A_{0}$, and the Jantzen region $J$ from Section 1. An element $\lambda \in X$ is called integral, or a weight, provided $\langle\lambda, \alpha\rangle \in \mathbb{Z}$ for all $\alpha \in R$. A weight $\lambda$ is called dominant (resp. regular dominant) if $\langle\lambda, \alpha\rangle \in \mathbb{Z}_{+}$(resp. $\mathbb{N}$ ) for all $\alpha \in R^{+}$. We assume henceforth that $p \geq h$; this is necessary and sufficient to ensure that $A_{0}$ contains a regular dominant integral weight (cf. [4, Part II, $6.2(10)]$ ). We have

$$
A_{0}=\left\{\lambda \in X \mid 0<\langle\lambda, \alpha\rangle \text { for all } \alpha \in \Delta,\left\langle\lambda, \alpha_{0}\right\rangle<p\right\}
$$

(cf. [3, Sec. 4.3]). Similarly

$$
\mathcal{C} \cap J=\left\{\lambda \in X \mid 0<\langle\lambda, \alpha\rangle \text { for all } \alpha \in \Delta,\left\langle\lambda, \alpha_{0}\right\rangle<p(p-h+2)\right\}
$$


by (1.1,1.2). Thus $\mathcal{C} \cap J$ is the expansion of $A_{0}$ by a factor $p-h+2$. In particular, $\operatorname{Vol}(\mathcal{C} \cap J)=(p-h+2)^{n_{0}} \cdot \operatorname{Vol}\left(A_{0}\right)$. Since, aside from a set of volume zero, $\mathcal{C} \cap J$ is the disjoint union of the alcoves $w A_{0}, w \in L$, and since $\operatorname{Vol}\left(w A_{0}\right)=\operatorname{Vol}\left(A_{0}\right)$, we obtain the following easy answer to Question 1.

Lemma 2.1. The cardinality of $L$ is $(p-h+2)^{n_{0}}$.

We now begin to work on Question 3; more generally, we wish to determine the maximum length of an alcove in any level $k$ of the dominant cone. Notice first that the answer is independent of the choice of $p$ - increasing $p$ simply expands the whole picture, and does not change either the level of an alcove $w A_{0}$ or the number of hyperplanes separating $w A_{0}$ from $A_{0}$ (since this is just $\ell(w)$ ). It will be convenient to choose $p$ so that there is a unique dominant integral weight (namely $\rho$ ) in $A_{0}$; then each alcove $w A_{0} \subset \mathcal{C}$ will contain a unique dominant integral weight, $w \rho$. We claim this happens when $p=h$. To see this, recall that $\langle\rho, \alpha\rangle=1$ for all $\alpha \in \Delta$, and also $h=\left\langle\rho, \alpha_{0}\right\rangle+1$. Thus $\rho \in A_{0}$, by (2.1). If $\lambda \neq \rho$ is regular dominant integral, then $\langle\lambda, \alpha\rangle \in \mathbb{N}$ for all $\alpha \in \Delta$, and $\langle\lambda, \beta\rangle>1$ for some $\beta \in \Delta$. This implies that $(\lambda, \alpha) \geq(\rho, \alpha)$ for all $\alpha \in \Delta$, while $(\lambda, \beta)>(\rho, \beta)$, which in turn implies that $\left\langle\lambda, \alpha_{0}\right\rangle>\left\langle\rho, \alpha_{0}\right\rangle$ (write $\alpha_{0}$ as a positive integer linear combination of the simple roots, observing that every simple root occurs in the highest short root). By integrality, $\left\langle\lambda, \alpha_{0}\right\rangle \geq\left\langle\rho, \alpha_{0}\right\rangle+1=h=p$, and thus $\lambda \notin A_{0}$. We set $\boldsymbol{p}=\boldsymbol{h}$ for the remainder of this article.

Fix $k \in \mathbb{Z}_{+}$, let $A$ be an alcove in $\mathcal{C}_{k}$, and let $\lambda \in A$. For $\alpha \in R^{+}$, the number of hyperplanes $H_{\alpha, i}(i \in \mathbb{Z})$ of type $\alpha$ separating $A$ and $A_{0}$ is $\lfloor\langle\lambda, \alpha\rangle / h\rfloor$. Thus our problem is to compute

$$
\max \sum_{\alpha \in R^{+}}\lfloor\langle\lambda, \alpha\rangle / h\rfloor,
$$

where the maximum is taken over all elements $\lambda \in \mathcal{C}_{k}$ with $\langle\lambda, \alpha\rangle \notin h \mathbb{Z}, \forall \alpha \in R^{+}$. Because the value of $\lfloor\langle\lambda, \alpha\rangle / h\rfloor$ is constant on each alcove $A$ (and each alcove contains an integral weight), we may restrict attention to the integral weights $\lambda \in$ $\mathcal{C}_{k}$. Thus the maximization in (2.3) may be restricted to those weights $\lambda$ satisfying

1. $k h<\left\langle\lambda, \alpha_{0}\right\rangle<(k+1) h$,

2. $\langle\lambda, \alpha\rangle \in \mathbb{N}$ for all $\alpha \in \Delta$, and

3. $\langle\lambda, \alpha\rangle \notin h \mathbb{N}$ for all $\alpha \in R^{+}$.

The actual determination of the maximum in (2.3) depends on explicit knowledge of the roots, and proceeds case-by-case. The roots and weights "naturally" occur in some $\mathbb{R}^{n}$ (usually $n=n_{0}$, but in some cases it is more convenient to take $n>n_{0}$ ); we shall use the notation $x=\left(x_{1}, \ldots, x_{n}\right)$ for an integral weight in $\mathbb{R}^{n}$. Write

$$
F(x)=\sum_{\alpha \in R^{+}}\lfloor\langle x, \alpha\rangle / h\rfloor,
$$

the function to be maximized.

Because of the discontinuities in $F$, it is difficult to maximize using "standard" techniques. Instead, our main tool is provided by the following lemma.

Lemma 2.2. Assume $F$ attains a maximum on $\mathcal{C}_{k}$ at $x$, where $x$ is integral and lies on none of the hyperplanes $H_{\alpha, i}$. Then $\left\langle x, \alpha_{0}\right\rangle \equiv-1(\bmod h)$ and $\langle x, \alpha\rangle \not \equiv-1$ $(\bmod h)$ for $\alpha_{0} \neq \alpha \in R^{+}$. 
Proof. First let $\alpha_{0} \neq \alpha \in R^{+}$, and suppose $\langle x, \alpha\rangle \equiv-1(\bmod h)$; for definiteness, write $\langle x, \alpha\rangle=i h-1$ for some $i \in \mathbb{Z}$. Then $s_{\alpha, i}(x)=x+\alpha \in \mathcal{C}$, which means that $x$ belongs to an alcove $A$ having $H_{\alpha, i}$ as one if its walls, and moreover $s_{\alpha, i} A$ and $A_{0}$ lie on opposite sides of $H_{\alpha, i}$. Thus $F\left(s_{\alpha, i} x\right)=F(x)+1$, but $s_{\alpha, i} x$ still belongs to $C_{k}$ (since the only wall that has been crossed is of type $\alpha$, and $\alpha \neq \alpha_{0}$ by assumption). This contradicts the hypothesis that $F(x)$ was a maximum value of $F$ in level $k$.

On the other hand, the alcove $A$ containing $x$ must have some wall $H$ such that reflecting $A$ across $H$ produces an alcove in $\mathcal{C}$ with length one greater. The only remaining possibility is that $H$ is of type $\alpha_{0}$; i.e., $\left\langle x, \alpha_{0}\right\rangle \equiv-1(\bmod h)$.

We have now reduced the problem to maximizing $F(x)$, subject to:

$$
\begin{aligned}
& \text { 1. }\left\langle x, \alpha_{0}\right\rangle=(k+1) h-1, \\
& \text { 2. }\langle x, \alpha\rangle \in \mathbb{N} \text { for all } \alpha \in \Delta, \\
& \text { 3. }\langle x, \alpha\rangle \notin h \mathbb{N} \text { for all } \alpha \in R^{+} \text {, and } \\
& \text { 4. }\langle x, \alpha\rangle \not \equiv-1(\bmod h) \text { for all } \alpha_{0} \neq \alpha \in R^{+} .
\end{aligned}
$$

In the classical cases, these conditions on $x$ are enough to determine $x(\bmod h)$ to within a handful of choices. We then analyze how to assign each $\left\lfloor x_{i} / h\right\rfloor$ (given $x$ $(\bmod h))$ in order to maximize $F$, subject to the constraints 1 and 2 . Finally, we choose the $x(\bmod h)$ which produces the largest value of $F$. In Sections 36 we implement the strategy just described for each classical irreducible root system in turn. Although the approach is unified, there are interesting subtle differences to each type of root system.

In the exceptional cases, there are longer lists of possible $x(\bmod h)$, and this strategy (while feasible, with the assistance of a computer in type $E_{n}$ ), is tedious. Instead, we adopt a different approach (suggested by the referee), which involves computing the generating function of dominant alcove lengths. (Both methods produced the same answers!) The generating function method is described and carried out in Section 7 .

\section{TYPE $A_{n-1}(n \geq 2)$}

Let $\varepsilon_{1}, \ldots, \varepsilon_{n}$ be the standard basis for $\mathbb{R}^{n}$, and ( , ) the standard inner product. We take the simple roots for $A_{n-1}$ to be the elements $\varepsilon_{i}-\varepsilon_{i+1}, 1 \leq i<n$. The positive roots are then the $\varepsilon_{i}-\varepsilon_{j}, 1 \leq i<j \leq n$. The highest root is $\alpha_{0}=\varepsilon_{1}-\varepsilon_{n}$, and $h=n$. Thus the conditions (2.5) on $x$ are

$$
\begin{gathered}
x_{1}-x_{n}=(k+1) n-1, \\
x_{i}-x_{i+1} \in \mathbb{N} \quad(1 \leq i<n), \\
x_{i}-x_{j} \not \equiv 0 \text { or }-1 \quad(\bmod n) \quad(i<j, \quad(i, j) \neq(1, n)) .
\end{gathered}
$$

Note that $\rho=\frac{1}{2}(n-1, n-3, \ldots, 1-n)$, and the weights $x$ we consider are of the form $w \rho=\rho-\sum_{i=1}^{n-1} n_{i}\left(\varepsilon_{i}-\varepsilon_{i+1}\right)$ with $n_{i} \in \mathbb{Z}$. It follows that the entries $x_{i}$ are all integers when $n$ is odd, and are all half-integers when $n$ is even.

Let's first consider $x(\bmod n)$. Since $x_{i}-x_{j} \not \equiv 0(\bmod n)$ for $i \neq j$, the $x_{i}$ $(\bmod n)$ are distinct. Since there are exactly $n$ possible distinct integer (resp. halfinteger) remainders modulo $n$, it follows from the Pigeonhole Principle that the $x_{i}$ $(\bmod n)$ are the $n$ distinct remainders. Moreover, using the condition $x_{i}-x_{j} \not \equiv-1$ $(\bmod n)$ for $i<j$ except when $(i, j)=(1, n)$, we see that the indicies $i, j$ associated 
with successive remainders (where we view 0 as succeeding $n-1$, and $\frac{1}{2}$ as succeeding $n-\frac{1}{2}$ ) must be decreasing except when $i=1, j=n$. This easily implies that

$$
x_{i}-x_{i+1} \equiv 1 \quad(\bmod n) \quad \text { for } 1 \leq i<n ;
$$

notice that this already implies the condition $x_{1}-x_{n} \equiv-1(\bmod n)$.

Now we need to determine $\left\lfloor x_{i} / h\right\rfloor$. Observe that

$$
F(x)=\sum_{1 \leq i<j \leq n}\left\lfloor\frac{x_{i}-x_{j}}{n}\right\rfloor,
$$

and set $F_{i j}^{-}(x)=\left\lfloor\left(x_{i}-x_{j}\right) / n\right\rfloor$. Set $m=\left\lfloor\frac{n}{2}\right\rfloor$. Recall from (3.1) that $x_{i} \geq x_{i+1}+1$. Suppose that $x_{r-1} \geq x_{r}+h+1$ for some $1<r \leq m$; in this situation we say that there is a gap between $x_{r-1}$ and $x_{r}$. Then $x_{r}$ can be increased by $h$ (or $x_{r-1}$ decreased by $h$ ) without affecting $x(\bmod h)$ (or any of the conditions in (3.1)). Define $x^{\prime}$ by

$$
x_{i}^{\prime}= \begin{cases}x_{i}+h, & i=r, \\ x_{i}, & i \neq r\end{cases}
$$

Now the sum of the coordinates of $x^{\prime}$ is no longer zero (as required in type $A$ ), but we can rectify that by defining $x^{\prime \prime}$ via $x_{i}^{\prime \prime}=x_{i}^{\prime}-1,1 \leq i \leq n$ (recall $h=n$ in type $\left.A_{n-1}\right)$. And since $F\left(x^{\prime \prime}\right)=F\left(x^{\prime}\right)$, we may as well work with the simpler $x^{\prime}$ instead of $x^{\prime \prime}$. We will compare $F\left(x^{\prime}\right)$ to $F(x)$. We have

$$
\begin{aligned}
& F_{i, r}^{-}\left(x^{\prime}\right)=F_{i, r}^{-}(x)-1, \quad i<r, \\
& F_{r, j}^{-}\left(x^{\prime}\right)=F_{r, j}^{-}(x)+1, \quad j>r,
\end{aligned}
$$

whence $F\left(x^{\prime}\right)=F(x)-(r-1)+(n-r)=F(x)+n+1-2 r>F(x)$ since $r<(n+1) / 2$. Similarly, if $x$ has a gap between $x_{r-1}$ and $x_{r}$ for $r>m+1$, then we obtain a larger (or equal) value of $F$ by decreasing $x_{r-1}$ by $n$. In particular, if $F(x)$ is the maximum value of $F$ on level $k$, we may assume that the only gap in $x$ is between $x_{m}$ and $x_{m+1}$. Using (3.1) and (3.2), we deduce that the maximum value of $F$ on level $k$ occurs at

$$
\tilde{x}=(a, a-1, \ldots, a-m+1, a-m-k n, a-m-k n-1, \ldots, a-(k+1) n+1)
$$

(where $a$ is chosen to make $\sum \tilde{x}_{i}=0$ ). It is easy to see that

$$
F(\tilde{x})=k m(n-m)=\left\{\begin{array}{ll}
k \frac{n^{2}}{4}, & n \text { even } \\
k \frac{n^{2}-1}{4}, & n \text { odd }
\end{array}\right\}=k\left\lfloor\frac{n^{2}}{4}\right\rfloor .
$$

Keeping in mind our shift from $n$ to $n-1$, this proves Theorem 1.1 in type $A$.

\section{Type $B_{n}(n \geq 2)$}

We take the simple roots for $B_{n}$ to be the elements $\varepsilon_{i}-\varepsilon_{i+1}, 1 \leq i<n$, together with $\varepsilon_{n}$; the positive roots are $\varepsilon_{i} \pm \varepsilon_{j}, 1 \leq i<j \leq n$, together with $\varepsilon_{i}, 1 \leq i \leq n$. The highest short root is $\alpha_{0}=\varepsilon_{1}$, and $h=2 n$. Thus the conditions on $x$ are:

$$
\begin{gathered}
x_{1}=(k+1) n-\frac{1}{2}, \\
x_{i}-x_{i+1} \in \mathbb{N} \quad(1 \leq i<n), \quad x_{n} \in \frac{1}{2} \mathbb{N}, \\
x_{i}-x_{j} \not \equiv 0 \text { or }-1 \quad(\bmod 2 n) \quad(i<j), \\
x_{i}+x_{j} \not \equiv 0 \text { or }-1 \quad(\bmod 2 n) \quad(i \leq j, \quad(i, j) \neq(1,1)) .
\end{gathered}
$$


Setting

$$
y=\left(x_{1}, \ldots, x_{n} ;-x_{n}, \ldots,-x_{1}\right),
$$

we see from (4.1) that the $y_{i}(\bmod 2 n)$ are $2 n$ distinct half-integers between 0 and $2 n$. By the Pigeonhole Principle, $\left\{y_{i}(\bmod 2 n)\right\}=\left\{\frac{1}{2}, \frac{3}{2}, \ldots, 2 n-\frac{1}{2}\right\}$. Moreover, $y_{i}-y_{j} \not \equiv-1(\bmod 2 n)$ when $i<j$ except for $(i, j)=(1,2 n)$, which forces $y \equiv$ $\left(2 n-\frac{1}{2}, 2 n-\frac{3}{2}, \ldots, \frac{3}{2}, \frac{1}{2}\right)$ or $\left(n-\frac{1}{2}, n-\frac{3}{2}, \ldots, \frac{1}{2} ; 2 n-\frac{1}{2}, \ldots, n+\frac{3}{2}, n+\frac{1}{2}\right)(\bmod 2 n)$. Thus $x \equiv\left(2 n-\frac{1}{2}, 2 n-\frac{3}{2}, \ldots, n+\frac{1}{2}\right)$ or $\left(n-\frac{1}{2}, n-\frac{3}{2}, \ldots, \frac{1}{2}\right)(\bmod 2 n)$, and the condition on $x_{1}$ in (4.1) implies that the former happens when $k$ is odd, the latter when $k$ is even.

For the "gap analysis," suppose that $x$ has a gap between $x_{r-1}$ and $x_{r}$; i.e., $x_{r-1} \geq x_{r}+h+1$ for some $1<r \leq n$. Define $x^{\prime}$ as in (3.3). Observe that

$$
F(x)=\sum_{1 \leq i<j \leq n}\left\lfloor\frac{x_{i}-x_{j}}{2 n}\right\rfloor+\sum_{1 \leq i \leq j \leq n}\left\lfloor\frac{x_{i}+x_{j}}{2 n}\right\rfloor,
$$

and set $F_{i j}^{ \pm}(x)=\left\lfloor\left(x_{i} \pm x_{j}\right) /(2 n)\right\rfloor$. Then

$$
\begin{array}{ll}
F_{i, r}^{-}\left(x^{\prime}\right)=F_{i, r}^{-}(x)-1, & i<r, \\
F_{r, j}^{-}\left(x^{\prime}\right)=F_{r, j}^{-}(x)+1, & j>r, \\
F_{i, r}^{+}\left(x^{\prime}\right)=F_{i, r}^{+}(x)+1, & i \neq r, \\
F_{r, r}^{+}\left(x^{\prime}\right)=F_{r, r}^{+}(x)+2, &
\end{array}
$$

whence $F\left(x^{\prime}\right)=F(x)-(r-1)+(n-r)+(n-1)+2=F(x)+2(n-r)+2>F(x)$. In particular, if $F(x)$ is the maximum value of $F$ on level $k$, then $x$ cannot have any gaps. But we know $x_{1}=(k+1) n-\frac{1}{2}$; combining this with our previously determined expressions for $x(\bmod 2 n)$, we find that the maximum of $F$ in level $k$ occurs at

$$
\tilde{x}=\left((k+1) n-\frac{1}{2},(k+1) n-\frac{3}{2}, \ldots, k n+\frac{1}{2}\right) .
$$

It is easy to compute $F(\tilde{x})=k\left(\begin{array}{c}n+1 \\ 2\end{array}\right)$. This completes the proof of Theorem 1.1 in type $B$.

\section{TYPe $C_{n}(n \geq 2)$}

We take the simple roots for $C_{n}$ to be $\varepsilon_{i}-\varepsilon_{i+1}, 1 \leq i<n$, together with $2 \varepsilon_{n}$; the positive roots are $\varepsilon_{i} \pm \varepsilon_{j}, 1 \leq i<j \leq n$, together with $2 \varepsilon_{i}, 1 \leq i \leq n$. The highest short root is $\alpha_{0}=\varepsilon_{1}+\varepsilon_{2}$, and $h=2 n$. Thus the conditions on $x$ are:

$$
\begin{gathered}
x_{1}+x_{2}=(k+1) 2 n-1, \\
x_{i}-x_{i+1} \in \mathbb{N} \quad(1 \leq i<n), \quad x_{n} \in \mathbb{N}, \\
x_{i}-x_{j} \not \equiv 0 \text { or }-1 \quad(\bmod 2 n) \quad(i<j), \\
x_{i}+x_{j} \not \equiv 0 \text { or }-1 \quad(\bmod 2 n) \quad(i<j, \quad(i, j) \neq(1,2)), \\
x_{i} \not \equiv 0 \text { or }-1 \quad(\bmod 2 n) \quad(1 \leq i \leq n) .
\end{gathered}
$$

Consider the remainders $\pm x_{i}(\bmod 2 n)$. These are all positive, and distinct except possibly $x_{i} \equiv-x_{i}(\bmod 2 n)$ for some $i$. But then $x_{i} \equiv n(\bmod 2 n)$, and this can happen for at most one $i$ (otherwise we'd have $x_{i}-x_{j} \equiv 0(\bmod 2 n)$ for some $\left.i<j\right)$. Defining $y=\left(y_{1}, \ldots y_{n} ; y_{n+1}, \ldots, y_{2 n}\right)$ as in (4.2) , the Pigeonhole Principle implies that $\left\{y_{i}(\bmod 2 n)\right\}=\{1, \ldots, 2 n-1\}$ with some $y_{t} \equiv y_{2 n+1-t} \equiv n(\bmod 2 n)(1 \leq$ $t \leq n)$, but with no other duplications among the $y_{i}(\bmod 2 n)$. Note also that $y_{i}$ 
$(\bmod 2 n)$ and $y_{2 n+1-i}(\bmod 2 n)$ are never consecutive integers, because they occur symmetrically with respect to $n$. Moreover, the conditions $y_{i}-y_{j} \not \equiv-1(\bmod 2 n)$ when $i<j$ except when $(i, j)=(1,2 n-1)$ or $(2,2 n)$, and $y_{i} \not \equiv-1(\bmod 2 n)$ when $i \leq n$ force $y(\bmod 2 n)$ to be one of the following:

$$
\begin{gathered}
{ }^{q} y=(2 n-q, q-1, q-2, \ldots, 2,1,2 n-q-1,2 n-q-2, \ldots, n+1, n ; \\
n, n-1, \ldots, q+2, q+1,2 n-1,2 n-2, \ldots, 2 n-q+1, q), \\
{ }^{q} \bar{y}=(q-1,2 n-q, q-2, \ldots, 2,1,2 n-q-1,2 n-q-2, \ldots, n+1, n ; \\
n, n-1, \ldots, q+2, q+1,2 n-1,2 n-2, \ldots, q, 2 n-q+1) .
\end{gathered}
$$

The first form is allowable for $1<q \leq n$, whereas the second (which is obtained from the first by interchanging $y_{1}$ and $y_{2}$ ) is allowable only for $1<q<n$, due to the condition $y_{1}-y_{2} \not \equiv-1(\bmod 2 n)$. Then $x(\bmod 2 n)$ must be one of

$$
\begin{aligned}
& (2 n-q, q-1, q-2, \ldots, 2,1,2 n-q-1,2 n-q-2, \ldots, n+1, n), \\
& (q-1,2 n-q, q-2, \ldots, 2,1,2 n-q-1,2 n-q-2, \ldots, n+1, n) .
\end{aligned}
$$

Anticipating the condition $x_{i}>x_{i+1}$, we set

$$
\begin{aligned}
{ }^{q} x= & (4 n-q, 2 n+q-1,2 n+q-2, \ldots, 2 n+2,2 n+1, \\
& \quad 2 n-q-1,2 n-q-2, \ldots, n+1, n), \quad 1<q<n, \\
{ }^{q} \bar{x}= & (4 n+q-1,4 n-q, 2 n+q-2, \ldots, 2 n+2,2 n+1, \\
& \quad 2 n-q-1,2 n-q-2, \ldots, n+1, n), \quad 2<q<n, \\
{ }^{n} x= & (n, n-1, n-2, \ldots, 2,1), \\
{ }^{2} \bar{x}= & (2 n+1,2 n-2,2 n-3, \ldots, n+1, n) .
\end{aligned}
$$

We have shown that if $F$ has a maximum on level $k$ at $x$, then $x \equiv{ }^{0} x(\bmod 2 n)$, where ${ }^{0} x$ is one of the weights in (15.2).

For the "gap analysis," assume first that $x$ has a gap beyond $x_{2}$; i.e., $x_{r-1}-x_{r}>$ $2 n$ for some $2<r \leq n$. Define $x^{\prime}$ as in (3.3); then $x^{\prime}$ is a legitimate parameter in the same level as $x$. In type $C_{n}$,

$$
F(x)=\sum_{1 \leq i<j \leq n}\left\lfloor\frac{x_{i}-x_{j}}{2 n}\right\rfloor+\sum_{1 \leq i<j \leq n}\left\lfloor\frac{x_{i}+x_{j}}{2 n}\right\rfloor+\sum_{1 \leq i \leq n}\left\lfloor\frac{x_{i}}{2 n}\right\rfloor .
$$

Thus $F\left(x^{\prime}\right)=F(x)-(r-1)+(n-r)+(n-1)+1=F(x)+2(n-r)+1>F(x)$. We deduce that if $F(x)$ is a maximum on level $k$, then $x$ has no gaps beyond $x_{2}$.

Next, suppose $x \in \mathcal{C}_{k}$ has a "double gap" between $x_{1}$ and $x_{2}$; i.e., $x_{1}-x_{2}>4 n$. Define $x^{\prime}$ by

$$
x_{i}^{\prime}= \begin{cases}x_{i}-2 n, & i=1, \\ x_{i}+2 n, & i>1,\end{cases}
$$

so that $x^{\prime} \equiv x(\bmod 2 n)$ and $x^{\prime} \in \mathcal{C}_{k}$ (recall that $x_{1}+x_{2}$ is constant on level $\left.k\right)$. In this case, $F\left(x^{\prime}\right)=F(x)-2(n-1)+2\left(\begin{array}{c}n-1 \\ 2\end{array}\right)-1+(n-1)=F(x)+n^{2}-4 n+2$, so that $F\left(x^{\prime}\right)>F(x)$ provided $n \geq 4$, whereas $F\left(x^{\prime}\right)<F(x)$ if $n=2,3$.

Thus, when $n \geq 4$, we obtain the largest value of $F(x)$ on $C_{k}$ for a given $x$ ( $\bmod 2 n)$ by taking $x_{1}$ (resp. $\left.x_{2}\right)$ as small (resp. large) as possible (subject to $x_{1}+x_{2}=(k+1) 2 n-1$ and $\left.x_{1}>x_{2}\right)$, and then taking $x_{i}(i>2)$ as large as possible (subject to $x_{i-1}>x_{i}>0$ ). When $n \leq 3$ we do exactly the opposite: 
choose $x_{1}$ as large as possible, and $x_{i}(i \geq 2)$ as small as possible, subject to the aforementioned constraints. More precisely, for $n \geq 4$, we must consider

$$
x={ }^{0} x+t \cdot 2 n(1, \ldots, 1)+\delta \cdot 2 n \varepsilon_{1},
$$

where ${ }^{0} x$ is one of the weights in (5.2), $t \in \mathbb{Z}_{+}$, and $\delta \in\{0,1\}$. When $n \leq 3$, we must consider

$$
x={ }^{0} x+t \cdot 2 n \varepsilon_{1} .
$$

It is easy to check that $F(x+t \cdot 2 n(1, \ldots, 1))=F(x)+t n^{2}$, and $F\left(x+t \cdot 2 n \varepsilon_{1}\right)=$ $F(x)+t(2 n-1)$. Also, if $x \in \mathcal{C}_{k}$ then $x+t \cdot 2 n(1, \ldots, 1) \in \mathcal{C}_{k+2 t}$ and $x+t \cdot 2 n \varepsilon_{1} \in \mathcal{C}_{k+t}$.

Now if $x=\left(x_{1}, \ldots, x_{n}\right)$ is either ${ }^{2} \bar{x}$ or ${ }^{q} x(2<q<n)$, then

$$
\bar{x}=\left(x_{2}, x_{1}, x_{3}, \ldots, x_{n}\right)+2 n \cdot \varepsilon_{1}
$$

(where ${ }^{2} \overline{\bar{x}}:={ }^{2} x$ ), with $\bar{x}$ one level above $x$, and $F(\bar{x})=F(x)+2 n-2$. Since $F(\bar{x})<F\left(x+2 n \varepsilon_{1}\right)$ (and both elements are in the same level), we may disregard weights of the form (5.3) or (5.4) with ${ }^{0} x={ }^{2} x$ or ${ }^{q} \bar{x}(2<q<n)$ when trying to maximize $F$.

For the remaining weights ${ }^{0} x$ in (5.2), we compute the data in the following table.

\begin{tabular}{c|c|c}
${ }^{0} x$ & Level & $F\left({ }^{0} x\right)$ \\
\hline${ }^{n} x$ & 0 & 0 \\
${ }^{2} \bar{x}$ & 1 & $\frac{1}{2} n^{2}-\frac{1}{2} n+1$ \\
${ }^{q} x(2<q<n)$ & 2 & $3 n-2+\left(\begin{array}{c}n-1 \\
2\end{array}\right)+\left(\begin{array}{c}q-1 \\
2\end{array}\right)$
\end{tabular}

Evidently $F\left({ }^{q} x\right)(2<q<n)$ is largest when $q=n-1$, with $F\left({ }^{n-1} x\right)=n^{2}-\frac{1}{2} n+1$.

Assume that $n \geq 4$. Then the possible maxima of $F(x)$ on levels 0 through 3 are among the values in the following table.

\begin{tabular}{c|c|c} 
Level & $x$ & $F(x)$ \\
\hline 0 & ${ }^{n} x$ & 0 \\
\hline 1 & ${ }^{2} \bar{x}$ & $\frac{1}{2} n^{2}-\frac{1}{2} n+1$ \\
1 & ${ }^{n} x+2 n \varepsilon_{1}$ & $2 n-1$ \\
\hline 2 & $n-1$ & $n^{2}-\frac{1}{2} n+1$ \\
2 & ${ }^{2} \bar{x}+2 n \varepsilon_{1}$ & $\frac{1}{2} n^{2}+\frac{3}{2} n$ \\
2 & ${ }^{n} x+2 n(1, \ldots, 1)$ & $n^{2}$ \\
\hline 3 & ${ }^{n-1} x+2 n \varepsilon_{1}$ & $n^{2}+\frac{3}{2} n$ \\
3 & ${ }^{2} \bar{x}+2 n(1, \ldots, 1)$ & $\frac{3}{2} n^{2}-\frac{1}{2} n+1$
\end{tabular}

(We used the computation on level 1 to avoid considering the translates of ${ }^{n} x+2 n \varepsilon_{1}$ to levels 2 and 3, etc.) Clearly the maximum value of $F$ on level $k$ is

$$
F\left({ }^{n} x+k n(1, \ldots, 1)\right)=\frac{k}{2} n^{2} \quad(k=0,2),
$$


and

$$
F\left({ }^{2} \bar{x}+(k-1) n(1, \ldots, 1)\right)=\frac{k}{2} n^{2}-\frac{1}{2} n+1 \quad(k=1,3) .
$$

Using (5.3), we find that these two formulas extend to arbitrary $k \in \mathbb{Z}_{+}$, for $k$ even or odd, respectively.

Now assume that $n \leq 3$. In a similar fashion, we find that the possible maxima of $F(x)$ on levels 0 through 2 are among the values in the following table.

\begin{tabular}{c|c|c} 
Level & $x$ & $F(x)$ \\
\hline 0 & ${ }^{n} x$ & 0 \\
\hline 1 & ${ }^{2} \bar{x}$ & $\frac{1}{2} n^{2}-\frac{1}{2} n+1$ \\
1 & ${ }^{n} x+2 n \varepsilon_{1}$ & $2 n-1$ \\
\hline 2 & ${ }^{n-1} x$ & $n^{2}-\frac{1}{2} n+1$ \\
2 & ${ }^{n} x+4 n \varepsilon_{1}$ & $4 n-2$
\end{tabular}

A little algebra shows that the maximum value of $F$ on level $k, 0 \leq k \leq 2$, is $F\left({ }^{n} x+k \cdot 2 n \varepsilon_{1}\right)=k(2 n-1)$. Using (5.4), we find that the same formula holds for arbitrary $k \in \mathbb{Z}_{+}$.

This completes the proof of Theorem 1.1] in type $C$.

$$
\text { 6. TYPE } D_{n}(n \geq 4)
$$

We take the simple roots for $D_{n}$ to be $\varepsilon_{i}-\varepsilon_{i+1}, 1 \leq i<n$, together with $\varepsilon_{n-1}+\varepsilon_{n}$; the positive roots are $\varepsilon_{i} \pm \varepsilon_{j}, 1 \leq i<j \leq n$. The highest root is $\alpha_{0}=\varepsilon_{1}+\varepsilon_{2}$, and $h=2(n-1)$. Thus the conditions on $x$ are:

$$
\begin{gathered}
x_{1}+x_{2}=2(k+1)(n-1)-1, \\
x_{i}-x_{i+1} \in \mathbb{N} \quad(1 \leq i<n), \\
x_{n-1}+x_{n} \in \mathbb{N}, \\
x_{i}-x_{j} \neq \equiv 0 \text { or }-1 \quad(\bmod 2(n-1)) \quad(i<j), \\
x_{i}+x_{j} \not \equiv 0 \text { or }-1 \quad(\bmod 2(n-1)) \quad(i<j, \quad(i, j) \neq(1,2)) .
\end{gathered}
$$

The second and third conditions imply that either all $x_{i}$ are integers, or all $x_{i}$ are half-integers. But since $\rho=(n-1, n-2, \ldots, 1,0)$ and $x=w \rho$ for some $w \in W_{p}$, it's clear that in fact all $x_{i} \in \mathbb{Z}$. The same two conditions imply that $x_{i} \in \mathbb{N}$ for $1 \leq i<n$, but $x_{n}$ may be $\leq 0$ (provided $\left.x_{n-1}+x_{n}>0\right)$. Given $x=\left(x_{1}, \ldots, x_{n}\right)$ satisfying (6.1), notice that $x^{-}=\left(x_{1}, \ldots, x_{n-1},-x_{n}\right)$ also satisfies (6.1). Moreover, since

$$
F(x)=\sum_{1 \leq i<j \leq n}\left\lfloor\frac{x_{i}-x_{j}}{2(n-1)}\right\rfloor+\sum_{1 \leq i<j \leq n}\left\lfloor\frac{x_{i}+x_{j}}{2(n-1)}\right\rfloor,
$$

we have $F\left(x^{-}\right)=F(x)$. Thus, without loss of generality, we may assume $x_{i} \in \mathbb{Z}_{+}$ for all $i$.

Proceeding as in the $C_{n}$ case, we find that the $\pm x_{i}(\bmod 2(n-1))$ are all distinct except that $x_{i} \equiv-x_{i} \equiv 0(\bmod 2(n-1))$ for some unique $i$, and $x_{j} \equiv-x_{j} \equiv n-1$ 
$(\bmod 2(n-1))$ for some unique $j$. This in turn leads to the following possibilities for $x(\bmod 2(n-1))$. The "minimal" (lowest level) weights are

$$
{ }^{q} x= \begin{cases}(2 n-1,2 n-4,2 n-5, \ldots, n, n-1,0), & q=1, \\ (4 n-q-4,2 n+q-3,2 n+q-4, \ldots, 2 n-1, & \\ 2 n-q-3,2 n-q-4, \ldots, n, n-1,0), & 1<q<n-1, \\ (n-1, n-2, \ldots, 1,0), & q=n-1 .\end{cases}
$$

If ${ }^{0} x=\left(x_{1}, \ldots, x_{n}\right)$ is either a ${ }^{q} x(1 \leq q \leq n-1)$, or (recursively) obtained from a $^{q} x$ via one (or more) of the operations in (6.4) below, then the following are also possibilities for $x(\bmod 2(n-1))$ :

$$
\begin{aligned}
& \overline{{ }^{0} x}={ }^{0} x+(n-1)(1, \ldots, 1), \\
& \widetilde{{ }^{0} x}=\left(x_{2}, x_{1}, x_{3}, \ldots, x_{n}\right)+2(n-1) \varepsilon_{1} \quad(q \neq 1), \\
& \widehat{{ }^{0} x}=\left(x_{1}, \ldots, x_{n-2}, x_{n}, x_{n-1}\right)+2(n-1)(1, \ldots, 1,0) \quad(q \neq n-1) .
\end{aligned}
$$

Remark 6.1. If ${ }^{0} x \in \mathcal{C}_{k}$, then $\overline{{ }^{0} x}, \widetilde{{ }^{0} x} \in \mathcal{C}_{k+1}$ while $\widehat{{ }^{0} x} \in \mathcal{C}_{k+2}$. Using (6.2), we find that $F\left(\overline{{ }^{0} x}\right)=F\left({ }^{0} x\right)+\left(\begin{array}{c}n \\ 2\end{array}\right)=F\left({ }^{0} x\right)+\frac{1}{2} n^{2}-\frac{1}{2} n, F\left(\widetilde{{ }^{0} x}\right)=F\left({ }^{0} x\right)+2 n-3$, and $F\left(\widehat{{ }^{0} x}\right)=F\left({ }^{0} x\right)+n^{2}-n-1$. Since $\frac{1}{2} n^{2}-\frac{1}{2} n>2 n-3($ recall $n \geq 4), F\left(\overline{{ }^{0} x}\right)>F\left(\widetilde{{ }^{0} x}\right)$, and since $2\left(\frac{1}{2} n^{2}-\frac{1}{2} n\right)>n^{2}-n-1, F\left(\overline{\overline{{ }^{x}}}\right)>F\left(\widehat{{ }^{0} x}\right)$. Thus for the purposes of maximizing $F$ on a given level, we may ignore the operations ${ }^{\sim}$ and $\widehat{~}$.

For the "gap analysis," assume first that $x$ has a gap beyond $x_{2}$; i.e., $x_{r-1}-x_{r}>$ $2(n-1)$ for some $2<r \leq n$. Define $x^{\prime}$ as in (3.3); then $x^{\prime}$ is a legitimate parameter in the same level as $x$. Using (6.2), we compute $F\left(x^{\prime}\right)=F(x)-(r-1)+(n-r)+$ $(n-1)=F(x)+2(n-r) \geq F(x)$. So we may assume that $x$ has no gaps beyond $x_{2}$.

Next, suppose $x$ has a gap between $x_{1}$ and $x_{2}: x_{1}-x_{2}>2(n-1)$. Define $x^{\prime}$ by

$$
x_{i}^{\prime}= \begin{cases}x_{i}-(n-1), & i=1, \\ x_{i}+(n-1), & i>1,\end{cases}
$$

so that $x^{\prime} \equiv \bar{x}(\bmod 2(n-1))$ (notation as in $\left.(\underline{6.4})\right)$, a legitimate parameter in the same level as $x$. Then $F\left(x^{\prime}\right)=F(x)-(n-1)+\left(\begin{array}{c}n-1 \\ 2\end{array}\right)=F(x)+\frac{1}{2}(n-1)(n-4) \geq F(x)$. Thus we may assume $x$ has no gaps at all. More precisely, in view of our earlier analysis of $x(\bmod 2(n-1))$, we need only consider $x={ }^{q} x+t(n-1)(1, \ldots, 1)$ for $1 \leq q \leq n-1$ and $t \in \mathbb{Z}_{+}$.

The only integral weight in level 0 is, of course, ${ }^{n-1} x=\rho$, and $F\left({ }^{n-1} x\right)=0$. In level 1 we have $F\left({ }^{1} x\right)=\frac{1}{2} n^{2}-\frac{3}{2} n+3$, and $F\left({ }^{n-1} x+(n-1)(1, \ldots, 1)\right)=$ $\left(\begin{array}{l}n \\ 2\end{array}\right)=\frac{1}{2} n^{2}-\frac{1}{2} n>F\left({ }^{1} x\right)$. Thus $\left(\begin{array}{l}n \\ 2\end{array}\right)$ is the maximum value of $F$ on level 1 (and we need not consider translates of ${ }^{1} x$ by multiples of $(n-1)(1, \ldots, 1)$ in higher levels). In level 2 we have (for $1<q<n-1) F\left({ }^{q} x\right)=\frac{1}{2} n^{2}+\frac{1}{2} n+\left(\begin{array}{l}q \\ 2\end{array}\right)-1$, which is maximized when $q=n-2: F\left({ }^{n-2} x\right)=n^{2}-2 n+2$. The other possibility is $F\left({ }^{n-1} x+2(n-1)(1, \ldots, 1)\right)=2\left(\begin{array}{c}n \\ 2\end{array}\right)=n^{2}-n>F\left({ }^{n-2} x\right)$. Thus the maximum value of $F$ on level 2 is $2\left(\begin{array}{l}n \\ 2\end{array}\right)$. Finally, we conclude that the maximum value of $F$ on any level $k>2$ must be attained at ${ }^{n-1} x+k(n-1)(1, \ldots, 1)$, and that maximum value is $k\left(\begin{array}{l}n \\ 2\end{array}\right)$.

This completes the proof of Theorem 1.1 in type $D$. 


\section{THE EXCEPTIONAL TYPES}

Recall that if $A$ is an alcove in the dominant cone $\mathcal{C}, \ell(A)$ denotes the length of $A$ : the number of hyperplanes separating $A$ from $A_{0}$. Set $L(A)$ to be the level of $A$, so that $A \subset \mathcal{C}_{L(A)}$. Similarly, if a weight $\lambda$ is in the dominant alcove $A$, put $\ell(\lambda)=\ell(A)$ and $L(\lambda)=L(A)$. Concretely (recall (2.3) $), \ell(\lambda)=\sum_{\alpha \in R^{+}}\lfloor\langle\lambda, \alpha\rangle / h\rfloor, L(\lambda)=$ $\left\lfloor\left\langle\lambda, \alpha_{0}\right\rangle / h\right\rfloor$. Let $t, z$ be indeterminates, and define the alcove length generating function

$$
g(t, z)=\sum_{A \subset \mathcal{C}} t^{\ell(A)} z^{L(A)} .
$$

Viewing $g(t, z)=\sum_{k \in \mathbb{Z}} g_{k}(t) z^{k} \in \mathbb{Z}[t][[z]]$, our problem is to find the degree of $g_{k}(t)$ for $k \in \mathbb{Z}_{+}$. It turns out that $g(t, z)$ can be calculated, using the fact that the affine Weyl group is the semidirect product of the Weyl group $W$ and the root lattice (cf. e.g. 3]).

Remark 7.1. It follows from the proof of Lemma 2.1 that $g_{k}(1)=(k+1)^{n_{0}}-k^{n_{0}}$, the number of alcoves in $\mathcal{C}_{k}$. By a result of Bott [1], $g(t, 1)=\prod_{1}^{n_{0}}\left(1-t^{e_{i}}\right)^{-1}$, where the $e_{i}$ are the exponents of $W$.

Assume for the moment that the root lattice $\mathbb{Z} R$ equals the weight lattice $P=$ $\{\lambda \in X \mid\langle\lambda, \alpha\rangle \in \mathbb{Z}$ for all $\alpha \in R\}$. Let $\omega_{1}, \ldots, \omega_{n_{0}}$ be the fundamental dominant weights (a basis for $P$ ) corresponding to some fixed ordering $\Delta=\left\{\alpha_{1}, \ldots, \alpha_{n_{0}}\right\}$, so $\left\langle\omega_{i}, \alpha_{j}\right\rangle=\delta_{i j}$. For $w \in W$, write $w \rho=\sum n_{i}^{w} \omega_{i}$, and set

$$
\lambda_{w}=w \rho+\sum_{i: n_{i}^{w}<0} h \omega_{i}
$$

Notice that $\lambda_{w}$ is a dominant weight, because $n_{i}^{w}=\left\langle w \rho, \alpha_{i}\right\rangle=\left\langle\rho, w^{-1} \alpha_{i}\right\rangle=\langle\rho, \alpha\rangle$ for some $\alpha \in R$, and $|\langle\rho, \alpha\rangle|$ is an integer between 1 and $\max _{\beta \in R}\langle\rho, \beta\rangle=h-1$. It follows analogously that, for any weight $\psi, \lambda_{w}+h \psi$ lies on none of the root hyperplanes $H_{\alpha, k}$; i.e., it lies in some alcove.

Recall that each dominant alcove $A$ contains a unique regular dominant integral weight $\lambda$, which in turn is obtained by applying a unique element of the affine Weyl group $W_{h}$ to $\rho$. By the semidirect product description of $W_{h}$, taking into account the scaling by $h$ and our assumption that $\mathbb{Z} R=P$, we have

$$
\lambda=w \rho+h \sum k_{i} \omega_{i}=\lambda_{w}+h \psi
$$

for some unique $w \in W$ and $k_{i} \in \mathbb{Z}$; here $\psi=\sum_{n_{i}^{w}>0} k_{i} \omega_{i}+\sum_{n_{i}^{w}<0}\left(k_{i}-1\right) \omega_{i} \in P$. In fact, we show that $\psi$ is dominant (though not necessarily regular). For $n_{i}^{w}>0$ : $n_{i}^{w}+h k_{i}>0 \Longrightarrow h k_{i}>-n_{i}^{w}>-h \Longrightarrow k_{i}>-1 \Longrightarrow k_{i} \geq 0$; while for $n_{i}^{w}<0$ : $n_{i}^{w}+h k_{i}>0 \Longrightarrow h k_{i}>-n_{i}^{w}>0 \Longrightarrow k_{i}>0 \Longrightarrow k_{i} \geq 1$. Conversely, to each $w \in W$ and each dominant weight $\psi$, there corresponds (via (7.3) and the last sentence of the previous paragraph) a unique dominant alcove $A$.

For $1 \leq i \leq n_{0}$ define $\ell_{i}=\ell\left(h \omega_{i}+\rho\right)$ and $L_{i}=L\left(h \omega_{i}+\rho\right)$.

Lemma 7.2. Let $\psi=\sum k_{i} \omega_{i}, k_{i} \in \mathbb{Z}_{+}, w \in W$, and set $\lambda=\lambda_{w}+h \psi$. Then $L(\lambda)=L\left(\lambda_{w}\right)+\sum k_{i} L_{i}$ and $\ell(\lambda)=\ell\left(\lambda_{w}\right)+\sum k_{i} \ell_{i}$.

Proof. Fix $\alpha=\sum a_{i} \alpha_{i} \in R^{+}$. Note that

$$
\lambda=w \rho+h \sum_{n_{i}^{w}>0} k_{i} \omega_{i}+h \sum_{n_{i}^{w}<0}\left(k_{i}+1\right) \omega_{i} .
$$


Thus

$$
\lfloor\langle\lambda, \alpha\rangle / h\rfloor=\lfloor\langle w \rho, \alpha\rangle / h\rfloor+\sum_{n_{i}^{w}>0} k_{i} a_{i}+\sum_{n_{i}^{w}<0}\left(k_{i}+1\right) a_{i},
$$

while

$$
\left\lfloor\left\langle\lambda_{w}, \alpha\right\rangle / h\right\rfloor=\lfloor\langle w \rho, \alpha\rangle / h\rfloor+\sum_{n_{i}^{w}<0} a_{i}
$$

and

$$
\lfloor\langle h \psi+\rho, \alpha\rangle / h\rfloor=\langle\psi, \alpha\rangle+\lfloor\langle\rho, \alpha\rangle / h\rfloor=\sum_{i} k_{i} a_{i},
$$

where we have used again the fact that $1 \leq\langle\rho, \alpha\rangle \leq h-1$. In particular, putting $\psi=\omega_{i}$ in (7.4), we have $\left\lfloor\left\langle h \omega_{i}+\rho, \alpha\right\rangle / h\right\rfloor=a_{i}$. Thus $\lfloor\langle\lambda, \alpha\rangle / h\rfloor=\left\lfloor\left\langle\lambda_{w}, \alpha\right\rangle / h\right\rfloor+$ $\sum_{i} k_{i}\left\lfloor\left\langle h \omega_{i}+\rho, \alpha\right\rangle / h\right\rfloor$. Taking $\alpha=\alpha_{0}$, or summing over all $\alpha \in R^{+}$, gives the desired results.

Proposition 7.3. When the root lattice equals the weight lattice, the alcove length generating function is given by

$$
g(t, z)=\sum_{w \in W} t^{\ell\left(\lambda_{w}\right)} z^{L\left(\lambda_{w}\right)} / \prod_{i=1}^{n_{0}}\left(1-t^{\ell_{i}} z^{L_{i}}\right) .
$$

Proof. By the lemma and the discussion preceding it,

$$
\begin{aligned}
g(t, z) & =\sum_{w \in W} \sum_{\substack{k_{i} \in \mathbb{Z}_{+}, 1 \leq i \leq n_{0}}} t^{\ell\left(\lambda_{w}\right)+\sum k_{i} \ell_{i}} z^{L\left(\lambda_{w}\right)+\sum k_{i} L_{i}} \\
& =\sum_{w \in W} t^{\ell\left(\lambda_{w}\right)} z^{L\left(\lambda_{w}\right)} \sum_{\substack{k_{i} \in \mathbb{Z}_{+}, 1 \leq i \leq n_{0}}} \prod_{i=1}^{n_{0}} t^{k_{i} \ell_{i}} z^{k_{i} L_{i}} \\
& =\sum_{w \in W} t^{\ell\left(\lambda_{w}\right)} z^{L\left(\lambda_{w}\right)} \prod_{i=1}^{n_{0}} \sum_{k_{i} \in \mathbb{Z}_{+}}\left(t^{\ell_{i}} z^{L_{i}}\right)^{k_{i}} \\
& =\sum_{w \in W} t^{\ell\left(\lambda_{w}\right)} z^{L\left(\lambda_{w}\right)} / \prod_{i=1}^{n_{0}}\left(1-t^{\ell_{i}} z^{L_{i}}\right) .
\end{aligned}
$$

In general, the root lattice is a sublattice of index $f$, say, in the weight lattice. Now for each dominant alcove $A$ there will be $f$ pairs $(w, \psi)$, with $w \in W$ and $\psi$ dominant integral, for which $\lambda=\lambda_{w}+h \psi \in A$. Thus the expression on the right hand side of (7.5) will equal $f \cdot g(t, z)$. But since we are only concerned with the highest power of $t$ occurring with each power of $z$, the scalar coefficient $f$ is irrelevant.

Our strategy then is to compute, for each exceptional Weyl group $W$, the data $\ell\left(\lambda_{w}\right), L\left(\lambda_{w}\right), w \in W$, and $\ell_{i}, L_{i}, 1 \leq i \leq n_{0}$. Using (7.5), it is then straightforward to read off the maximal alcove length in each level.

Example 7.4. The case of $\mathbf{G}_{\mathbf{2}}$. Here one finds that

$$
g(t, z)=\frac{1+\left(t+t^{2}+t^{3}\right) z+\left(t^{4}+2 t^{5}+t^{6}\right) z^{2}+\left(t^{7}+t^{8}+t^{9}\right) z^{3}+t^{10} z^{4}}{\left(1-t^{6} z^{2}\right)\left(1-t^{10} z^{3}\right)} .
$$


TABLE 3. Maximal $\ell\left(\lambda_{w}\right)$ for $\lambda_{w}$ in level $L$

\begin{tabular}{c|ccccccccccccccccc} 
L: & 0 & 1 & 2 & 3 & 4 & 5 & 6 & 7 & 8 & 9 & 10 & 11 & 12 & 13 & 14 & 15 & 16 \\
\hline$F_{4}$ & 0 & 9 & 21 & 30 & 41 & 50 & 61 & 69 & 77 & 85 & 86 & & & & & \\
$E_{6}$ & 0 & 15 & 30 & 44 & 58 & 72 & 86 & 97 & 108 & 119 & 120 & & & & & \\
$E_{7}$ & 0 & 26 & 51 & 76 & 100 & 124 & 148 & 172 & 196 & 218 & 240 & 262 & 284 & 301 & 318 & 335 & 336 \\
$E_{8}$ & 0 & 36 & 91 & 135 & 181 & 226 & 271 & 316 & 360 & 406 & 449 & 494 & 538 & 582 & 625 & 670 & 711 \\
&
\end{tabular}

TABLE 4. Fundamental weight data

\begin{tabular}{c|cc|cc|cc|cc} 
& \multicolumn{2}{|c|}{$F_{4}$} & \multicolumn{2}{|c|}{$E_{6}$} & \multicolumn{2}{|c|}{$E_{7}$} & \multicolumn{2}{|c}{$E_{8}$} \\
$i$ & $\ell_{i}$ & $L_{i}$ & $\ell_{i}$ & $L_{i}$ & $\ell_{i}$ & $L_{i}$ & $\ell_{i}$ & $L_{i}$ \\
\hline 1 & 22 & 2 & 16 & 1 & 34 & 2 & 92 & 2 \\
2 & 42 & 4 & 22 & 2 & 49 & 2 & 136 & 3 \\
3 & 30 & 3 & 30 & 2 & 66 & 3 & 182 & 4 \\
4 & 16 & 2 & 42 & 3 & 96 & 4 & 270 & 6 \\
5 & & & 30 & 2 & 75 & 3 & 220 & 5 \\
6 & & 16 & 1 & 52 & 2 & 168 & 4 \\
7 & & & & 27 & 1 & 114 & 3 \\
8 & & & & & & & &
\end{tabular}

What is the highest power of $t$ occurring in the coefficient of $z^{k}$ when this expression is expanded as a power series in $z$ ? When $k=3 \mathrm{~m}$, the highest exponent of $t$ is clearly $10 \mathrm{~m}$, coming from $1 \cdot 1 \cdot\left(t^{10} z^{3}\right)^{m}$. (The first factor represents a term from the numerator, the second a term from $1 /\left(1-t^{6} z^{2}\right)$, and the third a term from $1 /\left(1-t^{10} z^{3}\right)$.) When $k=3 m+1$ we obtain $10 m+3$ from $t^{3} z \cdot 1 \cdot\left(t^{10} z^{3}\right)^{m}$. And when $k=3 m+2$ the highest exponent of $t$ is $10 m+6$, which arises from both $t^{6} z^{2} \cdot 1 \cdot\left(t^{10} z^{3}\right)^{m}$ and $1 \cdot t^{6} z^{2} \cdot\left(t^{10} z^{3}\right)^{m}$. Thus the maximal length of an alcove in level $k$ of the dominant cone in type $G_{2}$ is $\lfloor(10 k) / 3\rfloor$.

Notice that in the numerator of $g(t, z)$, we do not care about the actual coefficient of $z^{j}$, but only the degree in $t$ of this coefficient. Thus we need only keep track of the highest length $\ell\left(\lambda_{w}\right)$ which occurs for each level $L\left(\lambda_{w}\right)$, as $w$ varies over $W$. In Table 3, we present this data for the remaining exceptional types. Except for $E_{8}$, the values for $\ell(w)$ and $L(w)$ were computed using a FORTRAN program written by the author. For $E_{8}$, the calculations were done by the computer algebra software GAP [7], combined with its accompanying Coxeter group package chevie, and an extension of chevie kindly provided by Frank Lübeck.

The lengths and levels for the regular dominant weights $h \omega_{i}+\rho$ are easy to calculate, and are presented in Table 4 (Labeling of the simple roots follows [2].)

Proceeding as in Example [7.4, we find that for $F_{4}$, when $k=2 m$, the maximum length in level $k$ is $22 m=11 k$, whereas when $k=2 m+1$, the maximum length is $22 m+9=11 k-2$. In $E_{6}$, the maximum length in level $k$ is $16 k$ for all $k$, and in $E_{7}$ it is $27 k$. In $E_{8}$, when $k=2 m$, the maximum length is $92 m=46 k$; for $k=2 m+3(m \geq 0)$ it is $92 m+136=46 k-2$; and for $k=1$, the maximum length is $36=46 k-10$. This completes the verification of Theorem 1.1] for the exceptional types. 


\section{ACKNOWLEDGMENTS}

I thank John Coleman for interesting me in computing the Kazhdan-Lusztig polynomials associated to the Lusztig Conjecture, which in turn led to the problem solved in this article. You have been a good mentor ever since my undergraduate days. I also thank my colleagues Lenny Chastkofsky, Joe Fu, Will Kazez, and Ted Shifrin for helpful discussions in the early stages of thinking about the problem. Finally, thanks to the referee for suggesting a shorter and more elegant method for handling the exceptional types.

\section{REFERENCES}

[1] R. Bott, An application of the Morse theory to the topology of Lie groups, Bull. Soc. Math. France 84 (1956), 251-281. MR 19:291a

[2] N. Bourbaki, Groupes et algèbres de Lie, Chs. 4, 5, 6, Éléments de Mathématique, Hermann, Paris, 1968. MR 39:1590

[3] J. E. Humphreys, Reflection Groups and Coxeter Groups, Cambridge studies in advanced mathematics vol. 29, Cambridge University Press, Cambridge, 1990. MR 92h:20002

[4] J. C. Jantzen, Representations of Algebraic Groups, Pure and Applied Math vol. 131, Academic Press, Orlando, 1987. MR 89c:20001

[5] G. Lusztig, Hecke algebras and Jantzen's generic decomposition patterns, Adv. in Math. 37 (1980), 121-164. MR 82b:20059

[6] - Some problems in the representation theory of finite Chevalley groups, Proc. Symp. Pure Math. 37 (1980), 313-317. MR 82i:20014

[7] M. Schönert et. al., GAP - Groups, Algorithms, and Programming, Lehrstuhl D für Mathematik, Rheinisch Westfälische Technische Hochschule, Aachen, Germany, fifth edition, 1995.

[8] W. Soergel, Conjectures de Lusztig, Sem. Bourbaki, Vol. 1994-5, Astérisque 237 (1996), Exp. 793, 75-85. MR 98f:20029

Department of Mathematics, University of Georgia, Athens, Georgia 30602-7403

E-mail address: brian@math.uga.edu 